subject. It may be stressed that some biological training is to-day an essential to any liberal culture and should be as much an obligatory part of a school curriculum as arithmetic.

One great merit of botanical study from the point of view of general education is that, if properly taught, it provides perhaps the best medium for training in accurate observation. Observation consists essentially of two separate processes, namely, seeing the object or phenomenon and the apprehension of what is seen. The visual perception of the good and bad observer may be alike adequate, but it is in the degree of their apprehension that they differ. To train such powers it is essential to check the accuracy of appreciation either by means of verbal description or graphic representation. The graphic method is clearly more suited to the adolescent mind, whose limited vocabulary and limited feeling and understanding of the nuances of meaning of words unduly restricts his verbal precision. Drawing, if regarded strictly as a statement of observed facts, offers the best means of such training, and botanical material, because of its well-defined organization, is peculiarly suited for this purpose.

[To be continued.]

\title{
Surface Action in Biology
}

A JOINT symposium including Sections A A (Mathematical and Physical Sciences), B (Chemistry) and I (Physiology) was held on this topic at the recent meeting at Nottingham of the British Association.

Since the development of the Langmuir trough in 1917, several new methods have been introduced into our armoury of weapons used for attacking the problems connected with film structure and film reactions. Of these, two have been developed in some detail and have provided us with numerous interesting and novel results, which appear to have far-reaching biological implications.

In the first method, a number of mono-layers of barium stearate are successively built up on a chromium-plated surface. Brilliant interference colours are observed when the film is viewed at a suitable angle in polarized light, conveniently obtained with the aid of a 'Polaroid' screen. The interference minima are so sharp that increments in thickness so small as 1.6 A. can be detected. If in the outer layer the divalent barium ions be replaced by ions of a higher valency, for example, thorium, the surface is now 'conditioned' in that it will adsorb monolayers of a variety of substances, for example, proteins or cholesterol. On these surfaces in turn adsorption of a second monolayer can take place, for example, digitonin on cholesterol but not on epicholesterol. It is found that many of these selective adsorptive reactions are highly specific.

By superimposing instead of a series of barium stearate monolayers, mixed films containing barium stearate and stearic acid are employed, the composite mixed membrane may be leached with stearic acid solvents, and a skeleton membrane of barium stearate remains. Such a skeleton can be refilled with various liquids, and the successful penetration followed by observing the changes in the refractive index of the layers. By sensitization of such a skeleton film and coating with a monolayer, it is evidently possible to examine the permeability of adsorbed monolayers to different solvents.

This optical method of examination provides us in the first place with an elegant means of determining whether adsorption has taken place or no, for example, in such reactions as the antibody antigen reaction. If in this case the surface reaction is indeed as specific as the bulk colloid reaction, it is evident that the method may well prove an agreeable substitute for the usual precipitation technique. In respect to sensitivity, we may note that one square centimetre (ample for optical examination) requires only $10^{-7} \mathrm{gm}$. for deposition. In the second place, the optical examination of skeleton membranes permits us to investigate both their permeability and ease of penetration by a variety of solvents. Since such penetration involves not only considerations of pore size but also of the extents of interaction between the penetrating molecular species and the material of the membrane, it is evidently a convenient method for obtaining information on each of these factors.

The second method of investigation involves determination of the force area characteristics, and the modification in the phase boundary potential caused by the presence of the film. Since in general terms the force area values give us information about the non-polar and the phase boundary potential provides us with knowledge about the polar portions of a complex organic molecule, it has been found possible to make fairly detailed analysis about the orientation of even complex film-forming systems. This applies especially to substances existing in what is termed the homalic state, that is, extended on the surface, such as are found in the protein or cellulosic monolayers. 
Since chemical action involves an alteration in the magnitude of the dipole moment of at least a portion of the reacting molecule, this method is found most convenient for following rates of reaction which may involve either an interaction between molecules in the film with those in one of the bulk phases, or interaction between molecules existing in the film itself. In this latter case, a more or less continuous polymeric film can be built up. Such polymeric films present interesting properties in respect to their elastic behaviour when solid and in their viscosity when liquid, and merit detailed examination.

Since in many cases the molecular orientation in the monolayer can be affected by a change in pressure exerted on the film, it is possible to examine the effects of molecular orientation in the kinetics of film reactions. Very remarkable changes in reaction velocity can be effected in some cases by a minute change in the surface tension ; furthermore, in photo-chemical reactions in films we can control the extinction coefficient and thus the quantum efficiency of surface photo-chemical action.

In addition to such enzyme and chemical reactions, it is also possible to examine those loose molecular associations frequently termed 'complexes' in some detail. We have already referred to the adsorption of digitonin by cholesterol, and similar reactions are involved with other glucosides such as saponin and even with much simpler molecules. Thus long-chain alcohols, especially secondary alcohols, and long-chain acids, form remarkably stable mixed films, although no ester formation is involved. These complexes involve interaction between both the polar head groups and also between the non-polar chain or ring systems of the reactants. Many of the polar head group interactions can be interpreted in terms of a hydrogen bond, but both dipole - dipole and dipole - ion interactions are also involved. The film technique also permits us to examine the mechanism of penetration of one molecular species into a monolayer of another, and here again both polar and non-polar portions of each molecular species are involved. It is also possible to examine the anchoring together of a number of molecules in the surface monolayer by a polymeric substance introduced into the substrate, for example, the polyphosphoric acid systems on calcium stearate or tannin on a protein.

The method reveals that these molecular associations involving free energy changes of the approximate order of $\Delta F=10,000$ cal. are extremely common in those relatively complex organic compounds met with in biological fluids, and it does not seem unreasonable to assume that they are actually present in living systems. For example, lipoids and proteins form lipoprotein films possessing interesting properties, and we might anticipate the presence of such lipoproteins in sera containing both proteins and lipoids. We might emphasize the remarkable degree of specificity obtainable not only by a change in the orientation of a polar group, but also by a similar change in orientation, for example, by movement of a double bond in the nonpolar portion of the molecule.

Whilst it is possible to examine the polar and non-polar interaction separately by this method, much further work is required before a definite statement as to the actual number of bonds and the individual bond energies involved can be made. This goal is important in that it will then permit us to calculate, as Eyring has pointed out, the absolute reaction rates. But it is at least interesting to note that in a protein 'complex' we are satisfied by this experimental technique that a large number of bonds are involved. The process of denaturation must involve, according to theory, the breaking of a large number to form the activated state for denaturation. ERIC K. RIDEAL.

\section{French Society of Chemical Industry}

\section{Seventeenth Congress in Paris}

$\mathrm{U}^{\mathrm{N}}$ DER the presidency of Sir Robert Mond, member of the Institut de France, the Société de Chimie Industrielle held its seventeenth Congress and at the same time celebrated the twentieth anniversary of its foundation by the late M. Paul Kestner, between the dates of September 26 and October 3. Arrangements made by various committees presided over by M. J. Gérard, M. J. Bougault and M. G.-J. Painvin, were appreciated by a large gathering of French chemists and about two hundred and fifty delegates from different countries, including more than twenty from Great Britain. British delegates bearing congratulatory addresses were sent by the Royal Society, the various chemical societies and the Institute of Metals.

After the proceedings had been opened in the large hall of the Maison de la Chimie by the UnderSecretary of State for Commerce, Sir Robert Mond gave an address on the evolution of the 